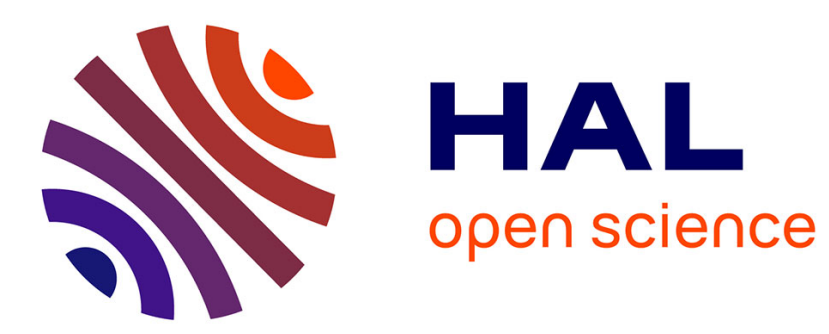

\title{
Effets de réseau dans la science pré-institutionnelle: le cas de l'optique médiévale
}

Dominique Raynaud

\section{To cite this version:}

Dominique Raynaud. Effets de réseau dans la science pré-institutionnelle: le cas de l'optique médiévale. Archives Européennes de Sociologie / European Journal of Sociology, 2001, 42 (3), pp.483-505. halshs00005535

\section{HAL Id: halshs-00005535 https://shs.hal.science/halshs-00005535}

Submitted on 13 Nov 2005

HAL is a multi-disciplinary open access archive for the deposit and dissemination of scientific research documents, whether they are published or not. The documents may come from teaching and research institutions in France or abroad, or from public or private research centers.
L'archive ouverte pluridisciplinaire HAL, est destinée au dépôt et à la diffusion de documents scientifiques de niveau recherche, publiés ou non, émanant des établissements d'enseignement et de recherche français ou étrangers, des laboratoires publics ou privés. 


\title{
Effets de réseau dans la science pré-institutionnelle: Le cas de l'optique médiévale
}

\author{
Dominique Raynaud ${ }^{1}$
}

Résumé : Cet article se demande pourquoi l'ordre franciscain, plus que d'autres organisations médiévales, religieuses ou laïques, prit part à la diffusion de l'optique. Des indices de cette asymétrie sociale sont tout d'abord donnés. Une explication est ensuite avancée: 1. Il existait une asymétrie initiale, caractérisée par le fait que l'optique de Grosseteste était connue au studium franciscain d'Oxford; 2. À partir de là, l'optique se diffusa au sein de l'ordre par un effet de réseau; 3. La rivalité entre les ordres mendiants et l'homophilie qui présidait à la politique d'acquisition de leurs bibliothèques conventuelles explique enfin pourquoi certaines organisations contemporaines, de structure comparable, ont porté moins d'intérêt à l'optique. En dépit d'une parenté thématique évidente, cette explication diffère profondément de l'analyse qui a été appliquée à l'activité scientifique des Puritains et des Jésuites dans l'Europe classique des XVIIe et XVIIIe siècles. Elle n'invoque pas l'hypothèse lourde de l'ethos pour comprendre le développement scientifique.

Mots-clefs : diffusion scientifique, réseaux sociaux, organisations religieuses.

Summary: This paper wonders why the Franciscan order took part in the diffusion of optics, more than other medieval organisation, both religious and secular. First, clues of this social asymmetry are given. Then, an explanation is put forward: 1 . An initial asymmetry existed, by the fact that Grosseteste's optics was known in the Franciscan studium of Oxford; 2. Since that date, optics spread among the order by a network effect; 3 . The rivaltry between the mendicant orders and the homophilia that presided over their conventual libraries' purchasing policy finally explains why other contemporary organizations, similarly structured, took a smaller interest into optics. In spite of an evident topical connection, this explanation deeply differs from the analysis that has been applied to the Puritans' and Jesuits' scientific activity in XVIIth and XVIIIth centuries classical Europe. It does not appeal to the heavy hypothesis of ethos in order to understand scientific development.

Key words: scientific diffusion, social networks, religious organizations.

\footnotetext{
${ }^{1}$ Université Pierre-Mendès-France, BP 47, 38040 Grenoble Cedex 9. Une première version de ce texte a été présentée au Séminaire d'histoire des sciences de l'antiquité à l'âge classique, organisé le 22 mai 1999 par le Centre d'histoire des sciences et des philosophies arabes et médiévales (GNRS UPRESA 7062, ÉPHÉ, Université de Paris 7). Je remercie Tony Lévy et Roshdi Rashed de m'avoir invité à participer à ce séminaire et d'avoir ainsi suscité une discussion entre historiens et sociologues.
} 


\section{Introduction}

Cet article, consacré à la diffusion des connaissances scientifiques dans un contexte antérieur à celui de la science institutionnelle du XVIIe siècle, suit le canevas de la sociologie comparative. À même de suggérer des explications de phénomènes relevant indifféremment du monde contemporain ou de l'histoire ancienne, ce modèle est un outil privilégié de la sociologie historique. Supposons que tous les éléments des deux situations comparées soient notés par les indices ${ }_{1}$ et ${ }_{2}$. Soit une action collective susceptible d'apparaître dans les groupes $G_{1}$ et $G_{2}$. La question initiale peut être formulée ainsi : qu'est-ce qui, dans les conditions initiales $\mathrm{C}_{1}$ et $\mathrm{C}_{2}$ de ces deux groupes, explique que les acteurs $\mathrm{A}_{1}$ ont pris part à l'action, alors que les acteurs $\mathrm{A}_{2}$ s'en sont abstenus ? Cette méthode de reconstruction rationnelle de singularités historiques peut être illustrée par un exemple.

Dans une étude consacrée aux types de communalisation religieuse, Max Weber se demande pourquoi les centurions et les fonctionnaires furent, plus que toute autre classe de la société romaine, attirés par les cultes de Mithra. La réponse se trouve dans l'examen de la nature de ces cultes. L'auteur écrit : « Le mithriacisme était une religion de la pureté, essentiellement ritualiste, uniquement réservée aux hommes (les femmes en étaient exclues, ce qui contraste fortement avec le christianisme). Il a été l'une des doctrines de salut les plus masculines [que l'on ait connue]. En outre il proposait toute une hiérarchie d'initiations et de grades religieux [...] L'attraction que ce culte a exercée sur les officiers tenait incontestablement [...] à l'avancement hiérarchique dans les degrés de l'initiation » (1995, 2 : 232-233). L'explication avancée par Max Weber est double : 1) Les centurions et les fonctionnaires se recrutant parmi les hommes, ils pouvaient adhérer à un culte exclusivement masculin ; 2) les centurions et les fonctionnaires étant rompus à une organisation hiérarchique, ils perçurent plus directement les bénéfices qu'ils pouvaient retirer d'une culte hiérarchisé. La démarche de Max Weber consiste ici à : 
1) sélectionner un phénomène qui paraît être une singularité historique irréductible ;

2) identifier les acteurs sociaux qui sont au cœur de cette singularité historique ;

3) percevoir dans la situation des acteurs, c'est-à-dire dans leur position ou leur disposition, le fondement de l'asymétrie sociale à expliquer (les centurions vs. les autres classes de la société romaine).

Cette méthode de réduction des singularités historiques est suffisamment générale pour être appliquée à la résolution de problèmes qui apparaissent dans le domaine de la sociologie historique des sciences. Cet article tente de cerner le rôle des facteurs organisationnels dans la diffusion des connaissances qui servirent de socle au développement de la perspective dans l'Italie du XVe siècle. Après avoir donné des indices de l'asymétrie qui fonde la singularité historique étudiée, il faudra identifier les acteurs sociaux impliqués, puis avancer une explication sociologique. Ce sera alors l'occasion de réexaminer un résultat classique de la sociologie des sciences, concernant le rôle des facteurs religieux dans le développement scientifique.

\section{Définition de l'asymétrie}

La question initiale pourrait être : «Pourquoi la contribution des Franciscains à la diffusion de l'optique médiévale fut-elle plus importante que celle d'autres organisations lä̈ques ou religieuses??. Si l'on veut parvenir à quelque certitude sur le rôle d'une organisation sociale impliquée dans la diffusion des connaissances scientifiques, il faut recourir à des méthodes présentant un caractère systématique $^{2}$. Construisons d'abord la base factuelle de l'asymétrie à partir de deux indicateurs du rôle des Franciscains : 1) la productivité scientifique en optique ; 2) la diffusion des traités d'optique.

\subsection{La productivité scientifique en optique}

\footnotetext{
${ }^{2}$ L'asymétrie énoncée s'applique à la diffusion macro-sociologique de l'optique. Elle ne suggère pas que tous les perspectivistes du Moyen Âge ont agi au sein, ou dans le sillage, de l'ordre franciscain. On sait en particulier que l'optique fut cultivée à la cour de Viterbe dans les années 1270 (Paravicini Bagliani, 1975). C'est dans le milieu de la cour pontificale que le chanoine Witelo composa sa Perspectiva et que son ami, le Dominicain Wilhelm de Moerbeke, rédigea une nouvelle traduction du De aspectibus d'Alhazen.
} 
On peut considérer que la forte productivité d'une organisation dans une discipline donnée est partiellement déterminée par la diffusion des connaissances à l'intérieur de l'organisation. L'étude de la productivité en optique nous renseigne donc indirectement sur l'état de diffusion interne de l'optique dans les ordres religieux du Moyen Âge. Classons, par nombre de traités, les titres du Catalogue of medieval and Renaissance optical manuscripts de Lindberg (1975) (Tableau I).

\begin{tabular}{|c|c|c|}
\hline Auteur & titres & nb. traités \\
\hline Robert Grosseteste [OFM] & De lineis, De luce, De iride, De motu, De colore, De natura locorum & 6 \\
\hline Roger Bacon OFM & Perspectiva, De mult. specierum, De speculis, De scient. experimentalis... & 6 \\
\hline John Pecham OFM & Perspectiva communis, Tractatus de perspectiva & 2 \\
\hline Ramon Llull OFM & Liber de lumine & 1 \\
\hline Bartolomeo da Bologna OFM & Tractatus de luce & 1 \\
\hline Geraldus de Odo OFM & Quaestio de lumine & 1 \\
\hline Henry de Langenstein OFM & Quaestiones super perspectivam & 1 \\
\hline Egidius de Baisiu OFM & Queritur causa quare lux transiens per foramen quadrangulare... & 1 \\
\hline Dietrich de Freiberg OP & De iride, De coloribus, De luce et eius origine & 3 \\
\hline Albert le Grand OP & Quaestio de forma resultante in speculo & 1 \\
\hline Thomas d'Aquin OP & De natura luminis & 1 \\
\hline Jean de Paris OP & De iride & 1 \\
\hline Witelo O.Can & Perspectiva & 1 \\
\hline Walter of Odington OSB & Tractatus de multiplicatione specierum in visu & 1 \\
\hline Nicole Oresme Sec & De visione stellarum, Quaestione de apparentia rei & 2 \\
\hline Thomas Bradwardine Sec & Propositiones de perspectiva & 1 \\
\hline Giovanni da Legnano DCL & De arbore consanguinitatis & 1 \\
\hline Domenico da Chivasso DMed & Quaestiones super perspectivam & 1 \\
\hline Dino del Garbo DMed & De visu & 1 \\
\hline Biagio Pelacani MA & Quaestiones super perspectivam & 1 \\
\hline Henry of Southwark MA & De visu et speculis & 1 \\
\hline Wigandus Durnheimer, inc. & Perspectiva & 1 \\
\hline Luca da Parma, inc. & Questio de visione & 1 \\
\hline Nicholas Bonetus, inc. & De lumine et colore & 1 \\
\hline
\end{tabular}

\section{Tableau I : Productivité comparée en optique ${ }^{3}$}

\footnotetext{
3 Nous avons retenu tous les traités d'optique vs. d'ophtalmologie, des XIIIe et XIVe siècles, portant un nom d'auteur. Nous n'avons pas entrepris de révision du catalogue car la recherche des manuscrits latins ne semble pas être parvenue au point où cette révision, toujours souhaitable, soit impérative. Mentionnons toutefois que Sturlese (1985) a proposé l'attribution de certaines notes d'optique au dominicain Berthold de Moosburg et que les travaux de Nikolaus von Dinkelsbühl, étudiés par Madre (1965), ne figurent pas dans ce catalogue.
} 
On constate que les Franciscains (OFM) ont composé 19 traités d'optique, alors que, dans le même temps, les Dominicains $(\mathrm{OP})$ ont rédigé 6 traités, les ordres canoniaux ou monastiques 2 traités, les séculiers 3 traités, les artiens, juristes et médecins 5 traités ; 3 de ces traités d'optique ayant été écrits par des auteurs mal connus.

\subsection{La diffusion des traités d'optique}

Un deuxième indice apparait lorsqu'on prend une mesure de la diffusion générale des traités. La recension des manuscrits — quoiqu'elle présente la difficulté d'être un outil de travail toujours provisoire - est en adéquation avec cette demande. Si l'on classe par nombre de manuscrits, les titres du Catalogue of medieval and Renaissance optical manuscripts de Lindberg (1975), on constatera que les traités d'optique les plus diffusés au Moyen Âge et à la Renaissance, sont encore ceux des Franciscains (Tableau II).

\begin{tabular}{|c|c|c|}
\hline Auteur & titres & mss \\
\hline Roger Bacon OFM & Perspectiva, De mult. specierum, De speculis, De scient. experimentalis... & 75 \\
\hline John Pecham OFM & Perspectiva communis, Tractatus de perspectiva & 72 \\
\hline Robert Grosseteste [OFM] & De lineis, De luce, De iride, De motu, De colore, De natura locorum & 58 \\
\hline Ramon Llull OFM & Liber de lumine & 6 \\
\hline Henry de Langenstein OFM & Quaestiones super perspectivam & 5 \\
\hline Bartolomeo da Bologna OFM & Tractatus de luce & 2 \\
\hline Geraldus de Odo OFM & Quaestio de lumine & 1 \\
\hline Egidius de Baisiu OFM & Queritur causa quare lux transiens per foramen quadrangulare... & 1 \\
\hline Albert le Grand OP & Quaestio de forma resultante in speculo & 16 \\
\hline Dietrich de Freiberg OP & De iride, De coloribus, De luce et eius origine & 8 \\
\hline Thomas d'Aquin OP & De natura luminis & 3 \\
\hline Jean de Paris OP & De iride & 1 \\
\hline Witelo O.Can & Perspectiva & 25 \\
\hline Walter of Odington OSB & Tractatus de multiplicatione specierum in visu & 1 \\
\hline Nicole Oresme Sec & De visione stellarum, Quaestione de apparentia rei & 6 \\
\hline Thomas Bradwardine Sec & Propositiones de perspectiva & 1 \\
\hline Biagio Pelacani MA & Quaestiones super perspectivam & 16 \\
\hline Giovanni da Legnano DCL & De arbore consanguinitatis & 5 \\
\hline Henry of Southwark MA & De visu et speculis & 2 \\
\hline Domenico da Chivasso DMed & Quaestiones super perspectivam & 1 \\
\hline Dino del Garbo DMed & De visu & 1 \\
\hline
\end{tabular}


$\begin{array}{ll}\text { Wigandus Durnheimer, inc. } \quad \text { Perspectiva } & 2\end{array}$

Luca da Parma, inc. Questio de visione 1

Nicholas Bonetus, inc. De lumine et colore 1

Tableau II : Diffusion des traités d'optique ${ }^{4}$

On constate que, sur un total de 310 mss., les mss. d'auteurs franciscains sont les plus nombreux (220 mss. soit 71\%), devant les Dominicains (28 mss. soit 9\%), les ordres canoniaux ou monastiques (26 mss. soit 8\%) et les autres catégories. C'est le signe de la large diffusion de ces textes et de l'intérêt qu'ils ont suscité5.

Ce résultat peut être affiné, sachant que les auteurs les mieux diffusés (Bacon et Pecham) ont intégré l'ordre respectivement ca. 1248 et 1251, qu’ils ont résidé au studium parisiense entre 1257 et 1267, et qu’ils ont l'un et l'autre étudié à Oxford sous Adam de Marsh, lui-même élève de Robert Grosseteste ${ }^{6}$. Les liens privilégiés entre ces auteurs suggèrent de réunir leurs textes sous une nouvelle catégorie : «optique des Franciscains issus d'Oxford ». Les items répondant à cette catégorie forment 205 mss. soit 66\%. En comparaison, la Perspectiva de Witelo, élaborée dans le milieu de la cour pontificale de Viterbe, a bénéficié avec 25 mss. d’une diffu-

\footnotetext{
4 Nous avons appliqué les mêmes critères qu'au tableau I.

5 Notons que, contrairement aux techniques de reproduction modernes, la copie d'un manuscrit est une tâche lourde, résultant toujours d'un engagement volontaire.

6 Robert Grosseteste fut lecteur au studium franciscain d'Oxford, de 1229 à 1235. Lié à Adam de Marsh, il protégea l'ordre mendiant et y encouragea l'étude des sciences naturelles. Matthieu de Paris affirme qu'il « fut toujours un extraordinaire imitateur de l'ordre, si bien qu'il prit la résolution de rejoindre leur ordre » [Semper ordinis aemulator singularis, adeo ut ad ordinem eorum propositum habuerit convolandi (Chronica maiora, IV, 599)]. Le projet n’aboutira pas, probablement en raison de l'âge avancé de Grosseteste.
} 
sion plus confidentielle que l'optique oxonienne ${ }^{7}$.

La superposition de ces indicateurs donne maintenant une lecture cohérente de l'asymétrie : aux XIIIe et XIVe siècles, les Franciscains ont contribué à la diffusion de l'optique plus que quelque autre organisation laïque ou religieuse, et le sociologue a toutes les raisons de suspecter le rôle de facteurs organisationnels.

\section{Interprétation sociologique}

Ayant donné une mesure de la diffusion des traités d'optique et de la productivité en optique, si l'on admet que la productivité dépend de la diffusion interne des connaissances au sein d'une organisation, on voit que les questions posées dans cet article se ramènent à l'étude du processus de diffusion interne et externe des connaissances. Le cadre conceptuel le plus adéquat pour traiter ce problème paraît être celui de la «sociologie des réseaux » (Wasserman et Faust, 1994 ; Degenne et Forsé, 1994), qui s’est développée à partir des travaux pionniers de Bavelas (1948) et de Flament (1965, 1968).

\footnotetext{
${ }^{7}$ Nous mentionnons ici des indicateurs de diffusion très généraux (nombre de traités, nombre de manuscrits). L'étude socio-historique de la diffusion de l'optique en Italie comme condition de développement de la perspective des peintres et des architectes corrobore ces résultats. Les traités de perspective de Leon Battista Alberti, Lorenzo Ghiberti, Piero della Francesca ou Leonardo da Vinci reproduisent des pans entiers des optiques de Bacon et de Pecham (Raynaud, 1998). Il est possible d'établir la marque de l'ordre franciscain non seulement sur les auteurs, mais aussi sur les messagers et les destinataires de ces textes. Concernant les messagers, Pecham dit à propos du Tractatus : «Scripsi dudum rogatus a sociis quedam mathematice ruditer rudimenta, que tamen aliis occupatus incorrecta reliqui, que etiam contra intentionem meam in publicum prodierunt » (Perspectiva communis, proemium). Or, au studium parisiense, Pecham eut pour étudiants Matteo d'Acquasparta et Pietro Giovanni Olivi, que l'on retrouvera l'un et l'autre en Italie à partir des années 1270. La présence des manuscrits d'optique de Santa Croce de Florence s'explique par le rôle de Pietro Giovanni Olivi à la tête du studium (1287-1289). Concernant les destinataires, la diffusion de l'optique de Pecham bénéficia de circonstances très favorables. Pecham doit son lectorat au Sacré-Palais à Jean XXI (Pierre d'Espagne), qui avait étudié la médecine à Paris, où il s'était lié avec Jean de Parme (alors ministre général des Franciscains) et l'anglais William of Sherwood, un proche de Bacon (Mann, 1964, $16: 33$ ). À la mort de Jean XXI (1277), Pecham eut l'opportunité de lui voir succéder Nicolas III (Giovanni Gaetano Orsini), dont le père appartenait au Tiers Ordre. Étant jeune, Giovanni avait connu personnellement saint François. Lors d'une rencontre, celui-ci lui confia la mission d'être un protecteur de l'ordre (Mann, 1964, 16 : 63). Ce qu'il fit, en devenant le cardinal protecteur des Franciscains à partir du règne de Clément IV. Le rayonnement de Pecham doit beaucoup à cette conjoncture favorable qui dura d'ailleurs jusqu'au début du XIVe siècle, puisque, de 1277 à 1302, la charge de lecteur au Sacré-Palais incombera exclusivement à des Franciscains. Or, c'est à cette époque que la perspectiva se diffusa en Italie.
} 
Les études de sociologie des réseaux portent généralement sur des terrains contemporains, car l'application à des phénomènes du passé pose quelques problèmes spécifiques : rares sont les cas où l'on parvient à reconstituer une information complète sur les relations qui composent un réseau social. On doit néanmoins saluer le travail de Padgett et Ansell (1993), qui ont trouvé la voie pour analyser les rapports entre les familles florentines du XVe siècle. Ces ouvertures sont exceptionnelles. L'étude des structures d'enseignement du Moyen Âge doit s'accommoder d'une situation moins propice, puisque les universités médiévales n'ont pas conservé de matricules avant le XVe siècle (Weijers et Holtz, 1997 : 20). Lorsque l’information est lacunaire, on peut : 1) soit recourir à des « données d'affiliation »;2) soit redéfinir des indicateurs sociologiques pertinents pour le phénomène à étudier. La première tactique offre l'avantage de préserver le calcul des indices mathématiques, mais, la base empirique étant sujette à caution, elle risque de donner une apparence de rigueur à des données incertaines. La deuxième tactique propose une application qualitative des modèles de la sociologie des réseaux. C'est la voie que nous suivrons dans la suite de cet article.

\subsection{Rappels de sociologie des réseaux}

Un réseau social se définit par l'ensemble des relations établies entre des acteurs ${ }^{8}$. Ces relations étant connues, on représente, en suivant les conventions de la théorie des graphes, les acteurs par des sommets et les relations par des arcs (si les relations sont orientées) ou par des arêtes (si les relations sont symétriques). La sociologie des réseaux tente alors de tirer des conclusions sociologiques d'une analyse structurelle de ces ensembles de relations.

La mesure de la densité du réseau (d) permet de distinguer : les réseaux cohésifs, qui ont pour modèle le «graphe complet »; les réseaux peu cohésifs, qui s'éloignent de ce modèle. Les réseaux peu cohésifs ont fait l'objet d'études particulières — «force des liens faibles » de Granovetter (1973), « hétérogénéité » de Blau (1977), «trous structuraux » de Burt (1992) —

\footnotetext{
${ }^{8}$ On distingue le réseau personnel, constitué par l'ensemble des relations d'une seule personne, du réseau complet qui tient compte, non seulement des relations interpersonnelles, mais également des relations transpersonnelles. Les remarques qui suivent concernent les réseaux complets.
} 
qui identifient quelques propriétés fondamentales. Dans un tel réseau, il existe souvent des sommets et des arcs faisant la jonction entre plusieurs composantes autonomes du réseau. Ce fait est à l'origine des concepts de point d'articulation (sommet dont le retrait entraîne la déconnexion du réseau) et de pont (arc ou arête dont le retrait entraîne la déconnexion du réseau). On définit le degré de connexité d'un graphe par le plus petit nombre d'arcs ou d'arêtes dont le retrait entraîne la déconnexion (Flament, 1965 : 51-52).

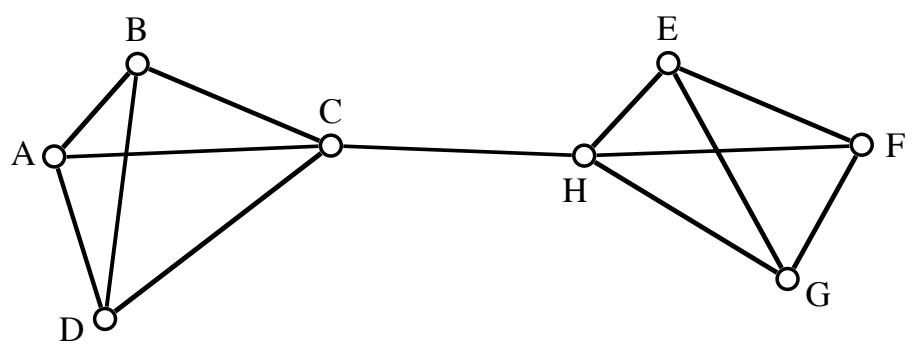

(1) $\delta=13 / 28=0,46$

1 pont $(\mathrm{CH}) ; 2$ points d'articulation $(\mathrm{C}, \mathrm{H})$

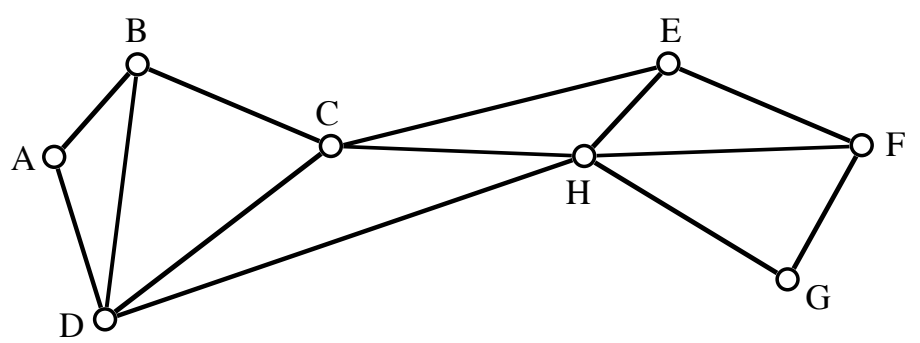

(2) $\delta=13 / 28=0,46$

3 arêtes $(\mathrm{CE}, \mathrm{CH}, \mathrm{DH})$; plus de point d'articulation

\section{Tableau III : Ponts et points d'articulation}

Prenons l'exemple des réseaux du Tableau III. Le degré de connexité du réseau de la figure (1), est $\mathrm{C}=1$. En effet, on peut identifier deux cliques $\mathrm{ABCD}$ et EFGH reliées par le pont $\mathrm{CH}$. On voit, sur cet exemple, que la densité n'est pas la seule caractéristique importante d'un réseau. En effet, quoique les réseaux (1) et (2) aient exactement la même densité $(\delta=0,46)$, ils n’ont pas les mêmes propriétés, n'ayant pas la même structure formelle. Dans le premier, il existe un seul pont entre ABCD et EFGH ; dans le second, ABCD et EFGH sont liés par trois arêtes.

Les psychosociologues ont tôt pressenti les conséquences de ces différences structurales : 
«En général, dans un groupe, un individu qui est un point d'articulation a par cela même, une position privilégiée ; assurant la liaison entre plusieurs sous-groupes, les relations, bonnes ou mauvaises, entre ces sous-groupes dépendent de lui » (Flament, 1968 : 56). Cette remarque est utile pour analyser la diffusion des connaissances. Supposons que A détienne une information et qu'il veuille l'adresser à F. Sur la figure 1 du Tableau III, si le pont CH est rompu l'information ne pourra plus passer entre les deux groupes ABCD et EFGH. En revanche, sur la figure 2, si CH est rompu, l'information pourra toujours circuler, soit par CE, soit par DH. On peut dire que, à densité comparable, la possibilité de transmission d'une information dépend de la structure du réseau. Cet exercice montre que les chances d'accéder à des informations nouvelles sont d'autant plus élevées que le groupe social possède davantage d'articulateurs.

La sociologie des réseaux permet de formaliser d'une façon élégante des résultats classiques de la sociologie des diffusions. Les travaux de Ryan et Gross (1943) ont établi - dans une étude portant sur l'adoption du maïs hybride - que les agriculteurs les plus cosmopolites sont les premiers à recourir à une innovation. Coleman, Katz et Menzel (1966) ont découvert un effet similaire dans la diffusion de nouveaux antibiotiques. Les médecins intégrés à de multiples institutions adoptent les innovations plus rapidement que leurs confrères isolés. Dans une veine un peu différente, Crozier et Friedberg (1977) ont aussi attiré l'attention sur le pouvoir des «marginaux-sécants » dans les organisations. Le marginal-sécant est cet «acteur qui est partie prenante de plusieurs systèmes d'action en relation les uns avec les autres et qui peut, de ce fait, jouer le rôle indispensable d'intermédiaire et d'interprète entre des logiques d'action différentes » (1977: 85). La situation particulière du marginal-sécant provient de ce qu’il craint moins l'exclusion du groupe, et que, en maîtrisant des segments sociaux que le groupe ne contrôle pas, il se rend indispensable à lui. Quel que soit leur point d'application, ces travaux montrent que la diffusion d'une information dépend étroitement des propriétés structurelles du réseau. Il existe, en ce sens, une équivalence entre les «cosmopolites », les « marginaux-sécants » et ce que la sociologie des réseaux appelle des « articulateurs ». On ver- 
ra maintenant que ces notions peuvent servir l'étude de la diffusion de l'optique médiévale.

\subsection{Les réseaux de l'optique}

L'asymétrie mise en évidence dans la première partie de cet article peut s'énoncer à divers niveaux de généralité. On peut se demander: pourquoi la contribution des Franciscains à l'optique médiévale est-elle plus importante que celle des Dominicains? Ou pourquoi cette contribution est-elle le fait d'un ordre mendiant versus d'un ordre canonial ou monastique? Ou bien pourquoi est-elle le fait du clergé régulier versus du clergé séculier? Et, à un niveau encore plus général, pourquoi est-elle le fait de clercs versus de laïcs? Ces questions, qui suivent le plan des institutions ecclésiastiques schématisé au Tableau IV, peuvent paraître d'un faible intérêt historique. Elles sont toutefois déterminantes pour conduire l'analyse sociologique. Je les reprendrai donc une à une.

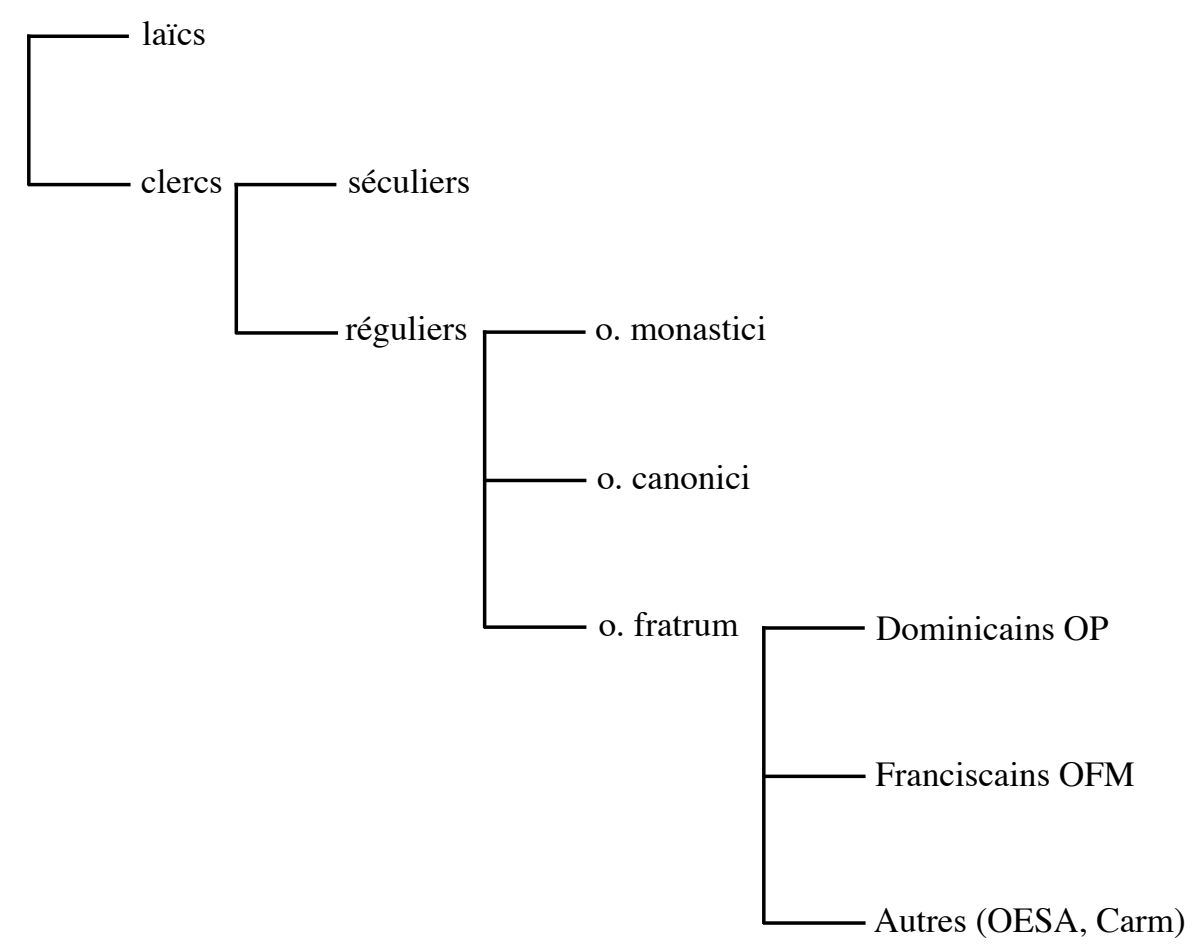

Tableau IV : Plan des institutions ecclésiastiques médiévales

2.2.1. Clercs / lä̈cs : L’opposition entre clercs et laïcs ne sollicite pas une enquête très approfondie. On sait en effet que l'université médiévale était une institution ecclésiastique. En at- 
teste l'ambiguïté du mot clericus qui signifie indifféremment : clerc versus laï, ou : instruit versus illettré. Revenant sur cette particularité de l'enseignement médiéval, Verger note qu'« on ne peut donc même pas parler de laïcs, au sens strict du mot, dans les universités médiévales, puisque tous les maîtres et étudiants, même laïques, bénéficiaient de privilèges cléricaux et relevaient de juridictions ecclésiastiques [...] De surcroît, la quasi-totalité des universitaires [...] restaient célibataires et recevaient des ordres, mineurs ou majeurs, ou, en tout cas, la tonsure ; cela leur permettait de jouir de la totalité des privilèges ecclésiastiques » (Verger, 1999 : 81). La laïcisation des structures universitaires sera surtout sensible à partir des XIVe et XVe siècles, à l'époque des créations princières. C'est à cette époque aussi que l'on y trouvera de plus en plus de maîtres et d'étudiants n'ayant pas reçu les ordres. La diffusion de l'optique des Grosseteste, Bacon et Pecham ayant eu lieu dans la deuxième moitié du XIIIe siècle, on comprendra qu'elle ait été entreprise par des clercs et non pas par des laïcs.

2.2.2. Séculiers / réguliers: Le facteur décisif partageant les deux clergés est que le clergé séculier a un statut territorial, alors que le clergé régulier a un statut extra-territorial. Le clergé séculier est placé sous l'autorité épiscopale, alors que « les religieux des ordres monastiques et mendiants sont soustraits à la juridiction de l'évêque diocésain »(Le Bras, 1980 : 261). On dit parfois qu'ils sont « exempts », pour signifier qu’ils dépendent directement de l'autorité pontificale. Ces éléments étant posés, on voit que la diffusion des connaissances ne pouvait tirer aucun parti d'une organisation territoriale. Pour que l'optique issue des milieux oxoniens passe dans d'autres régions européennes, il fallait au contraire qu'elle utilise une organisation extraterritoriale, pensée si possible à l'échelle de l'Occident chrétien. Le clergé régulier était donc mieux à même d'assurer cette diffusion que ne l'était le clergé séculier.

2.2.3. Ordres monastiques / mendiants : Il existe une différence entre les trois ordres qui composent le clergé régulier. La division s'opère entre ordo monasticus (comme les bénédictins et les cisterciens), ordo canonicus (comme les prémontrés et les chanoines réguliers de saint Augustin), ordo fratrum (comme les Dominicains et les Franciscains). L'opposition la plus nette apparaît entre les moines reclus, et les frères, connus pour leur esprit de mobilité. Comme dit Le Bras : 
«Tout au contraire des moines, qui cherchent le salut dans la solitude, vivent souvent à l'écart, voués à la contemplation, [...] les Mineurs s'engagent dans la foule, au service direct de l'église hiérarchique [...] La maison n'est pour eux qu'un abri de passage » (1964 : 459). Le statut des ordres canoniaux est intermédiaire entre les deux autres, pour au moins deux raisons : 1) la communauté de clercs est installée dans des maisons canoniales, dont l'église est ouverte au public ; 2) quoique exempts, les chanoines sont attachés à des tâches strictement diocésaines, donc territoriales. Les différents statuts des ordres réguliers laissent donc entrevoir une mobilité différentielle au sein des ordres monastiques (reclus), canoniaux (peu mobiles) et mendiants (très mobiles). Il suffit de se reporter au Tableau I, pour constater que la productivité en optique est directement corrélée à cette mobilité : les frères mendiants ont davantage contribué à l'étude de l'optique que les chanoines, les chanoines davantage que les moines ${ }^{9}$. La présence d'articulateurs susceptibles de prendre part à la diffusion des traités est une hypothèse économique pour expliquer ces différents niveaux de productivité scientifique.

2.2.4. Franciscains / Dominicains : Il reste enfin à expliquer pourquoi c'est au sein de l'ordre franciscain, et non au sein de l'ordre dominicain, que l'optique s'est développée, alors que les deux ordres mendiants présentaient exactement la même structure de groupes à articulateurs multiples ${ }^{10}$. L'hypothèse qu'on peut retenir est que cette asymétrie macroscopique développe une asymétrie initiale entre les deux ordres. Je reviendrai sur la nature de cette asymétrie initiale, avant d'examiner les raisons de son développement.

Une asymétrie initiale. Plusieurs historiens de l'optique (Crombie, 1953, Ten Doesschate,

\footnotetext{
${ }^{9}$ L'historien fait une observation concordante : « Les traditions de la culture monastique les tenaient [les moines] plutôt à l'écart des universités [...] Benoît XII avait bien essayé de donner aux réguliers traditionnels le goût des études théologiques en imposant aux couvents d'envoyer un moine sur vingt aux universités mais cette réforme échoua pratiquement devant la mauvaise volonté des abbés » (Verger, 1999 : 83, 112).

10 Cette limitation de l'analyse aux ordres Franciscain et Dominicain n'est pas arbitraire. On sait en effet que, étant les plus puissants des ordres mendiants, ils prirent une part prépondérante à l'étude. Le fait est souligné par Bacon : «Jamais il n’y eut de si belles manifestations de la science, ni un zèle si prononcé pour l'étude, en tant de facultés, ni en tant de pays, comme depuis quarante ans. Car partout on rencontre des docteurs [...] dans toutes les villes, toutes les places fortes, tous les bourgs, surtout grâce aux deux ordres qui étudient » [Ubique enim doctores sunt dispersi [...] in omni ciuitate et in omni castro et in omni burgo, praecipue per duos ordines studentes (Compendium studii philosophiae, I)].
} 
1962, Lindberg, 1995) ont suggéré que les travaux de Robert Grosseteste sur l'optique résultent d'une sensibilisation au sujet par une «métaphysique de la lumière », laquelle est clairement perceptible dans son traité De luce seu de inchoatione formarum. Crombie reconnaît ainsi que «L'analogie entre lux corporelle et lux spirituelle [...] doit être reconnue parmi les raisons qui assurèrent la popularité de l'optique et des sciences mathématiques à l'école d'Oxford » (1953 : 131). On ne devrait cependant pas surestimer le rôle de la métaphysique de la lumière, dans le cas de Bacon et de Pecham. Car les sept traités que Grosseteste a consacré à l'optique suffisent à constituer une asymétrie qui, par simple «effet d'orientation », a pu engager ses disciples directs ou indirects (comme Bacon et Pecham) dans l'étude de cette science (Raynaud, 2001, à paraître). L'accessibilité des travaux de Grosseteste au studium d'Oxford et l'enseignement d'Adam de Marsh suffisent donc à rendre compte de l'intérêt porté par leurs successeurs aux phénomènes optico-lumineux, et à expliquer pourquoi, à la fin du XIIIe siècle, on compte deux fois plus de traités franciscains que de traités dominicains (Tableau I).

Le développement de l'asymétrie. On se demandera maintenant pourquoi cette asymétrie initiale ne s'est pas estompée en quelques années entre les deux ordres mendiants, et pourquoi on la retrouve jusque dans les inventaires du XVe siècle des bibliothèques florentines (Raynaud, 1998 : 322-333). Des trois bibliothèques conventuelles de la cité, la bibliothèque dominicaine de Santa Maria Novella avait un seul texte relatif à l'optique (l'encyclopédie de Bartholomew of England, qui en expose les rudimenta). Celle des Augustins conservait un seul exemplaire de la Perspectiva de Witelo. La bibliothèque franciscaine de Santa Croce possédait au contraire sept manuscrits d'optique (dont deux assez rares : le De luce seu de inchoatione formarum de Grosseteste et le Tractatus de perspectiva de Pecham). L’asymétrie entre les ordres n’a donc pas disparue. Et il existe à cela une raison sociologique identifiable : c'est la forte émulation — sinon les antagonismes déclarés 11 - entre les deux ordres, qui s'étaient engagés dans des controverses multiples, relatives au voeu de pauvreté, au statut des doctrines augustiniennes et aristotéli-

11 On trouvera par exemple des extraits significatifs de la controverse, qui naquit en 1269 entre Franciscains et Dominicains d'Oxford, au sujet de la pauvreté, dans l'article de Little (1926 : 851). 
ciennes, etc. Les soupçons que porte Roger Bacon sur les compétences du dominicain Wilhelm de Moerbeke doivent sans nul doute être lues dans cet esprit ${ }^{12}$. On peut rendre compte de cet antagonisme en définissant une mesure d'échange des manuscrits entre les ordres religieux du Moyen Âge. Prenons le cas des bibliothèques florentines. Les inventaires d'époque étant connus, on peut faire correspondre, à chaque titre d'inventaire, l'obédience religieuse de l'auteur (ligne), et celle de la bibliothèque dans laquelle il est conservé (colonne) (Tableau V).

\begin{tabular}{lrrr} 
Ordre & Santa Croce $($ OFM $)$ & Santo Spirito (OESA) & S. Maria Novella (OP) \\
& 785 mss. & 577 mss. & 740 mss. \\
\hline OFM & 19,62 & 9,45 & 5,14 \\
OESA & 1,15 & 11,96 & 1,35 \\
OP & 7,13 & 11,83 & 26,89 \\
autres & 72,10 & 66,71 & 66,62 \\
\hline total & 100,00 & 100,00 & 100,00
\end{tabular}

Tableau V: Statistique des bibliothèques conventuelles de Florence (\%)

On note que, toutes disciplines confondues, les bibliothèques conventuelles avaient une politique d'acquisition largement favorable aux auteurs du même ordre. Ainsi, un lecteur de la bibliothèque dominicaine de S. Maria Novella n'avait que 5 chances sur 100 de pouvoir lire un auteur franciscain. Cette politique d'acquisition répond à un principe d'homophilie. Les auteurs franciscains étant sous-représentés chez les Dominicains, ceux-ci avaient d'autant moins de chances de pouvoir étudier l'optique. L’homophilie réduisait donc les possibilités d'échange entre les ordres et accentuait du même coup les différences culturelles entre les deux ordres. Une situation réelle n'étant jamais homogène, il a sans doute existé des articulateurs entre les deux organisations mendiantes, mais les échanges et les collaborations étaient alors limités à la sauvegarde des intérêts généraux de l'Église ou de l’Universitél3. Ces articulateurs étant peu

\footnotetext{
12 «Accidit tanta falsitas in eorum operibus [...] Omnes autem alii ignorauerunt linguas et scientias et maxime ille Willelmus Flamingus » (Opus tertium, II).

13 On sait ainsi que Arlotto da Prato (OFM) et Hugues de Billom (OP) furent taxateurs conjoints des maisons de l'Université de Paris en 1282 (Glorieux, 1934 : 119). Il est possible que, en recourant aux services de frères liés à des ordres rivaux, l’Université ait cherché principalement à préserver ses propres intérêts.
} 
nombreux, l'asymétrie initiale a été entretenue plutôt qu'effacée ${ }^{14}$.

Ces quelques observations sur le fonctionnement des institutions ecclésiastiques suffisent à élucider le mécanisme de la diffusion de l'optique. Le clergé régulier était mieux préparé que le clergé séculier à contribuer à cette transmission ; les ordres mendiants, mieux que les ordres canoniaux et monastiques; et les Franciscains, mieux que les Dominicains, pour des raisons qui sont cette fois purement conjoncturelles (si Grosseteste avait été lecteur au studium dominicain d'Oxford, les noms associés à l'optique médiévale seraient peut-être ceux de Dominicains).

2.2.5. Détermination des articulateurs franciscains. Gabriel Le Bras (1964: 460) fait observer que les couvents ${ }^{15}$ de mendiants abritaient de 30 à 50 religieux. C'est-là une moyenne, avec des chiffres inférieurs pour le début du XIIIe siècle et les villes reculées, des chiffres supérieurs pour le XVe siècle et les métropoles. On connaît le nombre de frères à Oxford par les tailles levées sur les couvents lors des visites royales. En 1227, on compte 70 Dominicains et 63 Franciscains. En 1317, 90 Dominicains et 84 Franciscains (Little, 1926 : 819-820). D’autres données peuvent être extraites du Tractatus de adventu d'Eccleston. En 1256, la province d'Angleterre comptait 49 couvents et 1242 frères, ce qui fournit une moyenne de 25 religieux par couvent. Ces chiffres sont assez concordants. Au XIIIe siècle, un couvent franciscain comptait environ 60 personnes s'il s'accompagnait d'un studium generale, comme à Oxford ou Paris. Il hébergeait environ 20 personnes, s’il était excentré. Telle est la taille des « cliques » auxquelles nous avons affaire. Il reste à évaluer les relations que ces cliques entretenaient entre elles. Divers critères peuvent être retenus pour identifier un articulateur dans un groupe. Le plus pratique est ici d'étudier la province d'origine des frères, une origine étrangère impliquant l'expé-

${ }^{14}$ La faible contribution des Dominicains s'explique par le fait qu'ils n'avaient pas accès aux connaissances nécessaires pour étudier l'optique. On raisonne ici sur les conditions d'accès à l'information et non sur les compétences : c'est le filtre entre deux réseaux sociaux qui constitue le noyau de l'explication sociologique.

15 La raison pour laquelle il est ici question des couvents, et non des facultés, est double. «D'abord, [les mendiants] ne s'associaient qu'à la faculté de théologie et refusaient de suivre les cours de la faculté des arts et d'y prendre leurs grades, prétendant assurer dans leurs propres écoles la formation en arts de leurs étudiants [...] Ensuite, à l'intérieur même de la faculté de théologie, ils continuaient à dépendre strictement de leur ordre » (Verger, 1999: 86). Sur les rapports entre studia et universités, cf. Verger (1978). 
rience de la mobilité géographique et l'appartenance à différents segments sociaux.

La mobilité des étudiants. Les Constitutions de Narbonne (1260) précisent que chaque province avait le droit d'envoyer deux étudiants au studium parisiense. Pour Oxford, une règle similaire sera adoptée au chapitre de Milan (1285). Ces données suggèrent une mobilité assez faible. Toutefois, Little (1926 : 818) a montré que cette règle ne concernait que les étudiants de debito (ceux qui étaient aux frais du studium destinataire). Les étudiants de gratia n'étant pas comptés, parce qu'ils restaient à la charge des provinces d'origine. D'autres éléments attestent au contraire d'une forte mobilité. Il existait notamment une règle limitant le nombre de grades qu'un studium pouvait accorder aux ressortissants de sa propre province. Au chapitre de Milan (1285), il sera convenu que « la province de France ne peut présenter un de ses religieux, pour le baccalauréat ou le doctorat, qu'une année sur trois : les deux autres années étant réservées à l'ensemble des autres provinces » (Glorieux, 1934: 10). Si l'on suit cette indication à la lettre, le studium parisiense devait accueillir environ 2/3 d'étudiants étrangers.

La mobilité des enseignants. L’apparition de la licencia ubique docendi, au XIIIe siècle, témoigne d'une universalisation des grades délivrés par les studia generalia. Cette mesure eut une conséquence pratique, car elle permit aux étudiants et aux lecteurs de se translater d'un studium à un autre en conservant leurs titres et leurs fonctions. Les agents impliqués dans la diffusion de l'optique ont tous vécu cette mobilité géographique : John Pecham a enseigné à Oxford, Paris et Rome ; Matteo d'Acquasparta, à Paris, Bologne et Rome ; Pietro Giovanni Olivi, à Paris, Florence et Montpellier. Dans la carrière d'un lecteur de quelque réputation, trois studia semblent être une donnée courante. On peut également évaluer la mobilité des lecteurs ${ }^{16}$ à partir des registres des maîtres en exercice aux XIIIe et XIVe siècles. Ils permettent d'évaluer la proportion des lecteurs étrangers sur les chaires. Le calcul, effectué sur les trois principaux studia generalia franciscains du Moyen Âge (Oxford, Paris, Bologne) sur la période 1230-1350 montre

\footnotetext{
16 Nous préférons ici le terme de lecteur (lector). Le nom de magister est d'un usage plus délicat, car il peut désigner un maître actu regens ou non actu regens. De plus, chez les mendiants, le professeur pouvait ne pas avoir pris ses grades, rendant alors impropre l'usage du titre de magister (Weijers et Holtz, 1997 : 173).
} 
que le taux de cosmopolitisme du studium oxoniense était compris entre $5 \%$ et $9 \% 17$. Celui du studium parisiense était compris entre $63 \%$ et $65 \% 18$. Celui du studium bononiense, entre 67 et $76 \% 19$. En dépit de ces incertitudes, on parvient à une conclusion relativement claire. Dans la période 1230-1350, le taux moyen de cosmopolitisme sur ces trois studia était de 47\% (soit environ un lecteur étranger pour un lecteur autochtone), ce cosmopolitisme étant par ailleurs plus sensible sur les provinces centrales que sur les provinces périphériques ${ }^{20}$.

Les principaux articulateurs des studia franciscains des XIIIe et XIVe siècles furent donc les étudiants étrangers et les lecteurs étrangers. À Paris, les uns et les autres représentaient près des 2/3 de leurs effectifs respectifs. Ce cosmopolitisme élevé était, à l'évidence, une condition de diffusion des connaissances que n’offraient pas les autres formes de clergé régulier. Les monastères constituaient des cliques fermées, les maisons canoniales des cliques semi-fermées. Seuls les couvents de mendiants (Franciscains ou Dominicains) répondaient au modèle de groupes à articulateurs multiples. Les remarques faites sur la circulation des informations dans un réseau, sont appliquables ici : par leur forte articulation sur l'extérieur, les ordres mendiants (versus les ordres canoniaux et monastiques) constituaient un instrument de prédilection pour la diffusion des sciences, et notamment de l'optique médiévale.

${ }^{17}$ Le Tractatus de adventu de Thomas d'Eccleston fournit une liste des lecteurs qui se succédèrent au studium d'Oxford. Si l'on étudie les origines des 74 lecteurs ayant exercé de 1229 à 1345 (67 lecteurs mentionnés par Thomas +4 lecteurs séculiers +1 lecteur oublié), on trouve que 68 lecteurs étaient originaires d'Angleterre, 4 issus d'une province étrangère, 2 d'origine douteuse ou inconnue - calcul d'après Little (1926) et Emden (19571959). L'écart entre 5\% et 8\% s'explique par l'incertitude de plusieurs notices prosopographiques. Hormis les difficultés classiques (Verger, 1988), les causes d'incertitude sont ici que le lecteur est parfois désigné par son seul prénom (Iohannes lector), que le toponyme est trop répandu (Robertus de Sancta Cruce), ou que le lieu n'est pas identifiable (Thomas Stanshaw).

1843 lecteurs ont exercé la charge de régent au studium de Paris, de 1231 à 1320. On y dénombre 15 lecteurs de la province de France, 28 étrangers, 1 d'origine inconnue — calcul d'après Glorieux (1934, 1951).

1942 lecteurs ont exercé la charge de régent à Bologne, entre 1223 et 1369. Parmi eux, on dénombre 10 lecteurs de la province de Bologne, 28 étrangers et 4 d'origine inconnue - calcul d'après Piana (1970, 1986).

20 On trouve là une indication très claire, non seulement de la direction du courant de diffusion de l'optique médiévale, mais aussi des phénomènes d'hybridation qui ont pu se produire dans cette région d'Italie centrale (tel que le développement de la perspective des peintres et des architectes). Plus un studium est cosmopolite, plus il attire à lui des lecteurs étrangers et plus il accède à des connaissances nouvelles. 


\section{Protestants, Jésuites, Franciscains}

Le rapprochement de certains travaux de sociologie, concernant le rapport entre les facteurs religieux et le développement scientifique, fait apparaître une question sur laquelle il convient de s'arrêter avant de conclure. Dans une étude classique de sociologie des sciences — qui est aussi la première du genre - Robert K. Merton (1970) a établi que le développement des sciences dans l'Angleterre du XVIIe siècle fut indissociable de l'ascension du puritanisme. Dans un volume récent, les coauteurs réunis par Luce Giard (1995), ont tenté de montrer la forte implication des Jésuites dans la production scientifique des XVIIe et XVIIIe siècles. Il semble qu'on puisse maintenant identifier une connexion du même type entre les Franciscains et l'optique du XIIIe siècle. Le fait est assez récurrent pour que l'on questionne de façon détaillée ce rapport entre les organisations religieuses (Puritains, Jésuites, Franciscains) et les pratiques scientifiques. Revenons tout d'abord aux deux premières thèses.

\subsection{L'investissement scientifique des Puritains}

Merton (1970) cherche à comprendre pourquoi, dans les années 1670, l'élite anglaise a manifesté un intérêt pour les sciences expérimentales, en prenant part, notamment, aux activités de la Royal Society fondée en 1660. L’analyse de notices prosopographiques montre que cette élite de savants était principalement composée de Puritains. Le canevas de l’interprétation mertonienne est que : a) une religion se traduit par l'adhésion à un ensemble de valeurs ; b) la science devient socialement déterminante à partir de son institutionnalisation ; c) l'ethos puritain (pragmatisme, utilitarisme, libre arbitre) valorisait, à l'échelle collective, les qualités requises pour les pratiques scientifiques d'orientation expérimentaliste. L'éthique protestante, parce qu'elle était en rupture avec la tradition religieuse des siècles passés, aurait facilité l'innovation scientifique. Cette thèse a suscité diverses mésinterprétations, déjà pointées par Abraham (1983) et Ben-David (1991), qui tiennent généralement à une simplification du mo- 
dèle de causalité réellement utilisé par le fondateur de la sociologie des sciences ${ }^{21}$. Robert K. Merton s'applique en effet à percevoir une relation d'« interdépendance fonctionnelle » entre science et puritanisme. Il écrit par exemple : «Il pourrait sembler que je prenne la religion comme variable indépendante et la science comme variable dépendante durant cette période, bien que $[\ldots]$ ce ne soit pas du tout dans mon intention [...] Il faut simplement admettre qu'à la fois le puritanisme et la science ont été des composants d'un système complexe de variables mutuellement dépendantes » $(1973: 248)^{22}$.

\subsection{L'investissement scientifique des fésuites}

Dans l'ouvrage collectif sur Les fésuites à la Renaissance (Giard, 1995), le chapitre rédigé par Steven Harris présente un grand intérêt du point de vue sociologique. L’auteur montre que les mathématiques pures et appliquées ont constitué l'essentiel de la production scientifique des Jésuites de 1540 à 1773, dates respectives de la fondation et de la suppression de la Compagnie (1995 : 247-251). Ce lien étant mis en évidence, l’interprétation bute sur un obstacle que l'auteur identifie spontanément. Steven Harris reconnaît en effet que « si les graphiques et les décomptes d'auteurs et de publications tracent les contours généraux de l'activité mathématique des Jésuites, ils ne nous disent pas comment s'est produit l'investissement dans cette discipline » (1995: 250). L'éthique des Jésuites peut-elle expliquer cet intérêt scientifique? L’article n'apporte pas de réponse significative, mais l'introduction générale de l'ouvrage offre un indice sur le rapport de ces recherches à la thèse mertonienne : «L’ironie des choses a fait

\footnotetext{
${ }^{21}$ Cf. aussi la correspondance Sorokin-Merton, dans laquelle on trouve notamment une discussion sur la causalité, datée de juin 1934, où Pitirim A. Sorokin fait part à son étudiant de ses doutes sur le modèle de la causalité efficiente : «it is definitely over » (Merton 1989 : 295). Ces extraits de correspondance ont été produits par Merton, à l'occasion du séminaire international "Fifty years on the Merton thesis" (Tel Aviv / Jérusalem, 16-19 mai 1988).

22 Et c'est pourquoi son étude envisage aussi l'influence rétroactive de la science sur le puritanisme : « Le témoignage de la science ne manque pas de corroborer les croyances religieuses. La conviction d'une nature mutuellement confirmatoire de la raison et de la révélation offrait une nouvelle base à l'attitude favorable envers les études expérimentales, lesquelles ont, c'est admis, renforcé les dogmes théologiques fondamentaux » (1973 : 243, souligné par moi).
} 
qu'une étude à la gloire de la Réforme [...] aura préparé le retour sur la scène historique d'un ordre religieux actif dans la Contre-Réforme » (Giard 1995 : lii). C'est en fait dans une autre publication de l'auteur (Harris 1989) que l'on trouve une tentative explicite de transposition de la thèse mertonienne aux Jésuites. Après une démonstration pas-à-pas, qui revient notamment sur toutes les difficultés théoriques soulevées par la thèse mertonienne (1989 : 32-37), l'auteur conclut à une « confirmation paradoxale de la thèse de Merton » (1989 : 60). Paradoxale, en effet, car en montrant que la «spiritualité apostolique », noyau dur de l'éthique de la Compagnie de Jésus, a favorisé le développement des sciences, on doit admettre que : 1) la Contre-Réforme et la Réforme, c'est-à-dire le parti de la tradition et celui de la rupture, offraient des systèmes de valeurs antagonistes ; 2) l'ethos discontinuiste de la Réforme a favorisé l'innovation scientifique ; 3) l'ethos continuiste de la Contre-Réforme a également favorisé l'innovation scientifique. L'auteur propose une issue à ce paradoxe en supposant que l'intérêt scientifique a, dans les deux cas, été conditionné par le résidu commun de l'expérience des Puritains et des Jésuites, à savoir qu'ils constituaient des «groupes idéologiquement motivés » (1989 : 60), ce qui revient à admettre le rôle secondaire des contenus spécifiques de ces éthiques respectives.

\subsection{Ethos et réseau social}

Les difficultés soulevées par le rapprochement de ces travaux incitent donc à se garder d'une extrapolation des thèses de Merton au cas franciscain. Sans vouloir renoncer totalement aux analyses en termes d'ethos — qui s'avèrent souvent pertinentes — peut-être l'étude des causes du développement scientifique devrait-elle partir des données les plus immédiates, avant d'invoquer l'hypothèse lourde de l'ethos religieux. Car, si les Puritains, les Jésuites et les Franciscains se caractérisent bien par une éthique spécifique, ils se définissent aussi, et peut-être avant tout, en tant qu'organisations religieuses.

Les sociologues ont tendance à décrire les organisations par leur morphologie; il en est de même des organisations religieuses. Dans les Institutions ecclésiastiques de la Chrétienté médiévale, 
Gabriel Le Bras (1964) explicite les rapports qui lient le frère au gardien, le gardien au ministre provincial, le provincial au ministre général. Il décrit avec beaucoup de soin le fonctionnement des divers chapitres conventuel, provincial et général. Mais toute organisation conditionne, nolens volens, le développement de relations qui ne suivent pas les découpages morphologiques. Il y a donc, en toute organisation, deux objets sociologiques confondus : l'organisation proprement dite, considérée comme morphologie (laquelle se caractérise par le type de pouvoir, les modalités de décision collective, etc.) et le réseau social formé par les relations sociales singulières tissées entre les acteurs de cette organisation sociale. Quoiqu'on ait tendance à identifier des morphologies là où il n'y a pas de réseaux — et des réseaux là où il n'y pas de morphologie - Jean Baechler a raison de dire qu'« il ne faudrait pas les concevoir comme imperméables l'un à l'autre » (1992: 79). En effet, on peut souvent appliquer ces deux points de vue à la description des mêmes entités. Dans le cas présent, c'est manifestement le réseau associé à l'organisation franciscaine, et non sa morphologie, qui explique la contribution des frères à l'optique.

\section{Conclusion}

L'étude de la productivité scientifique et de la diffusion de l'optique au sein de l'ordre franciscain rentre dans le sillage immédiat des travaux de Harris, qui faisait remarquer : « Ce que je trouve surprenant à propos de [la] littérature [sur la thèse mertonienne] est combien peu elle se préoccupe d'étendre le programme de recherche historique de Merton » (1989: 29), et recommandait, en conclusion, de considérer que « Les sujets les plus appropriés pour continuer de telles recherches comparatives sont précisément là, où nous n’avons pas jugé bon de regarder auparavant, nommément, au sein d'autres ordres religieux catholiques » (1989:61). L'étude de l'optique franciscaine satisfait à ces conditions. Toutefois, elle ne conduit pas à une confirmation pure et simple du rôle moteur de l'éthos religieux. Elle met plutôt en lumière un 
aspect souterrain et beaucoup plus fondamental de la thèse de Merton ${ }^{23}$, qui concerne la structure du réseau social au sein duquel se déploie l'activité scientifique. Le résultat auquel nous parvenons peut se résumer en quelques propositions de synthèse :

1) Une des vocations essentielles de toute organisation scientifique est de diffuser des connaissances (car cette diffusion stimule en retour la production scientifique).

2) L’optique médiévale ne pouvait pas bénéficier du réseau de diffusion caractéristique de la science institutionnelle qui naît au XVIIe siècle.

3) Dans un contexte, qui est donc celui de la science pré-institutionnelle, la diffusion a été assurée par des réseaux sociaux qui ne poursuivaient pas, a priori, d'objectif scientifique. On pourrait parler ici de réseau substitutif ou plus exactement de détournement, à des fins scientifiques, d'un réseau à vocation religieuse.

En définitive, on constate qu'il n'est pas toujours utile de mobiliser la notion d'ethos pour comprendre l'investissement dans une discipline scientifique, pas plus qu'il n'est utile de mobiliser des facteurs religieux pour rendre compte du développement des sciences, y compris lorsque celui-ci utilise les ressources sociales d'une organisation religieuse. Les facteurs organisationnels suffisent parfois à rendre compte de tels phénomènes. En retour, il conviendrait de reprendre l'étude des « effets de réseau » au sein des milieux anglais et continentaux, car cela constitue une piste de recherche prometteuse pour ramener l'antagonisme apparent des valeurs réformistes et contre-réformistes à une solution élégante.

23 En ce qui concerne le développement des sciences expérimentales au XVIIe siècle, il serait intéressant de reprendre l'étude des réseaux de la Royal Society et des Philosophical Transactions. Merton s'approche de certains résultats de la sociologie des réseaux lorsqu’il écrit : «Les éditeurs des Transactions étaient membres de la Royal Society, qui était en relation directe avec les scientifiques de premier plan » (1973: 202) ; « Comme on sait bien, les universités étaient le siège du conservatisme et d'une négligence presque totale de la science, plutôt qu'un vivier de la nouvelle philosophie. Ce furent surtout les sociétés savantes qui produisirent l'association et l'interaction sociale des scientifiques, avec de tels résultats remarquables. Les Philosophical Transactions, puis d'autres journaux, supplantèrent grandement le mode de communication des idées scientifiques par la correspondance personnelle, qui prévalait antérieurement et était insatisfaisant » (1973: 247). 


\section{Bibliographie}

Abraham, Gary A. (1983). Misunderstanding the Merton thesis: A boundary dispute between history and sociology, Isis $74: 368-387$.

Alhazen (Ibn al-Haytham al-Hasan), Opticae thesaurus Alhazeni Arabi libri septem. Eiusdem liber de crepusculis et nubium ascensionibus... a Federico Risnero, Basilae, anno 1572.

Bacon, Roger (1964). The Opus maius of Roger Bacon, edited with introduction and analytical table by J.H. Bridges, Frankfurt am Main, Minerva GmbH [1266].

Baechler, Jean (1992). Groupes et sociabilité, Boudon R., éd., Traité de sociologie, Paris, PUF : 57-96.

Bavelas, Alex (1948). A mathematical model for group structures. Applied Anthropology 7 : 16-30.

Ben-David, Joseph (1991). Scientific growth. Essays on the social organization and ethos of science, Berkeley-London, University of California Press.

Blau, Peter (1977). Inequality and heterogeneity, New York, The Free Press.

Burt, Ronald S. (1992). Structural holes: The social structure of competition, Cambridge, Harvard University Press.

Coleman, James S., Katz, E. et Menzel, H. (1966). Medical innovation: A diffusion study, New York, Bobbs-Merrill.

Grombie, Alistair G. (1953). Robert Grosseteste and the origins of experimental science (1100-1700), Oxford, Clarendon Press.

Grozier, Michel et Friedberg, Ehrard (1977). L'acteur et le système, Paris, Éditions du Seuil.

Degenne, Alain et Forsé, Michel (1994). Les réseaux sociaux, Paris, Armand Colin.

Eccleston, Thomas d' (1909). Tractatus de adventu Fratrum Minorum in Anglia, edited by A.G. Little, Paris, Fischbacher [1258-1259].

Emden, Alfred B. (1957-1959). A biographical register of the University of Oxford to A.D. 1500, 3 vols, Oxford, Clarendon Press Ltd.

Flament, Claude (1965). Réseaux de communication et structures de groupe, Paris, Dunod.

Flament, Glaude (1968). Théorie des graphes et structures sociales, Paris, Gauthier-Villars.

Giard, Luce, éd. (1995). Les fésuites à la Renaissance. Système éducatif et production du savoir, Paris, PUF.

Glorieux, Palémon (1934). Répertoire des maîtres en théologie de Paris au XIIIe siècle, 2 vols, Paris, J. Vrin.

Glorieux, Palémon (1951). Maîtres franciscains régents à Paris. Mise au point, Recherches de Théologie ancienne et médiévale $18: 324-332$.

Granovetter, Mark S. (1973). The strenght of weak ties, American Fournal of Sociology 78 : 1360-1380.

Harris, Steven J. (1989). Transposing the Merton thesis : apostolic spirituality and the establishment of the Jesuit scientific tradition, Science in Context, "After Merton": Protestant and Catholic science in seventeenth century Europe, 3, 1 : $29-65$.

Harris, Steven J. (1995). Les chaires de mathématiques, Giard, L., éd. Les Jésuites à la Renaissance. Système éducatif et 
production du savoir, Paris, PUF : 239-261.

Le Bras, Gabriel (1964). Institutions ecclésiastiques de la Chrétienté médiévale, Bruxelles, Bloud \& Gay.

Le Bras, Gabriel, éd. (1980). Les ordres religieux, Paris, Flammarion.

Lindberg, David C. (1975). A catalogue of medieval and Renaissance optical manuscripts, Toronto, The Pontifical Institute of Medieval Studies.

Lindberg, David C. (1995). Medieval science and its religious context, Osiris 10 : 61-79.

Little, Andrew G. (1926). The Franciscan school at Oxford in the thirteenth century, Archivum Franciscanum Historicum 19 : 803-874.

Madre, Alois (1965). Nikolaus von Dinkelsbühl, Leben und Schriften. Beiträge zur Geschichte der Philosophie un Theologie des Mittelalters, 40 (4). Münster: Äschendorffsche Verlagbuchhandlung.

Mann, Horace K. (1964). The lives of the Popes in the Middle Ages, vols 14-18 (1243-1304), Vaduz, Kraus Reprint.

Merton, Robert K. (1970). Science, technology and society in 17th century England, New York, H. Fertig [1938].

Merton, Robert K. (1973). The sociology of science : Theoretical and empirical investigations. Chicago : University of Chicago Press.

Merton, Robert K. (1989). The Sorokin-Merton correspondence on puritanism, pietism and science, 1933-1934, Science in Context, "After Merton": Protestant and Catholic science in seventeenth century Europe, 3, 1 : 291-298.

Padgett, John F. et Ansell, Christopher K. (1993). Robust action and the rise of the Medici, 1400-1434, American Journal of Sociology 98 : 1259-1319.

Paravicini Bagliani, Agostino (1975). Witelo et la science optique à la cour pontificale de Viterbe (1277), Mélanges de l'Écçole française de Rome, Moyen Âge / Temps modernes 87-2 : 425-453.

Pecham, John (1970). Fohn Pecham and the science of Optics: Perspectiva communis, edited with an introduction, English translation and critical notes by D.C. Lindberg, Madison, Wisconsin University Press.

Piana, Gelestino (1970). Chartularium studii Bononiensis S. Francisci (saec. XIII-XVI). Ad Claras Aquas-Florentiae: Ex Typographia Collegii S. Bonaventurae.

Piana, Celestino (1986). Postille al "Chartularium Studii Bononiensis S. Francisci", Archivum Franciscanum Historicum, 79: 78-141, 449-499.

Raynaud, Dominique (1998). L'Hypothèse d'Oxford. Essai sur les origines de la perspective, Paris, PUF.

Raynaud, Dominique (2001). Optique et photisme au studium oxoniense : un éclaircissement, Pris-Ma (sous presse).

Roest, Bert (2000). A history of Franciscan education (c. 1210-1517). Leiden, E.J. Brill.

Rogers, Everett M. (1979). Network analysis of the diffusion of innovations, Holland, P.W., Leinhardt S., eds., Perspectives on social network research, New York, Academic Press : 137-164.

Ryan, Bryce et Gross, N.C. (1943). The diffusion of hybrid seed corn in two Iowa communities, Rural Sociology 8 : $15-24$. 
Sturlese, Loris (1985). Note su Bertoldo di Moosburg O.P., scienzato e filosofo, Freiburger Zeitschrift fir Philosophie und Theologie Freiburg, 32 (1/2): 249-259.

Valente, Thomas W. (1995). Network models of the diffusion of innovations, Creskill, Hampton Press.

Verger, Jacques (1978). Studia et universités. Le scuole degli ordini mendicanti, secoli XIII-XIV. Convegno del Centro di studi sulla spiritualità medievale, 11-14 ottobre 1976. Todi, Accademia Tudertina, 173-203.

Verger, Jacques (1988). Peut-on faire une prosopographie des professeurs des universités au Moyen Âge ? Mélanges de l'École française de Rome / Moyen Âge, 100 : 55-62.

Verger, Jacques (1999). Les Universités au Moyen Âge, Paris, PUF.

Wasserman, Stanley et Faust, Katherine (1994). Social network analysis: Methods and applications. Cambridge, Cambridge University Press.

Weber, Max (1995). Économie et société, 2 : L'organisation et les puissances de la société dans leur rapport avec l'économie, Paris, Plon [1956].

Weijers, Olga et Holtz, Louis, éds. (1997). L'enseignement des disciplines à la Faculté des arts (Paris et Oxford, XIIIe-XVe siècles), Actes du colloque international, Paris, 18-20 mai 1995, Turnhout, Brepols. 